\title{
Atividade fotossintética de plantas cultivadas em solo contaminado com
}

\section{sulfentrazone ${ }^{1}$}

\section{Photosynthetic activity of cultivated plants in soil contaminated with sulfentrazone}

\author{
Alessandra Ferreira Belo ${ }^{2}$, Lino Roberto Ferreira ${ }^{3}$, Evander Alves Ferreira ${ }^{4}$, Luciana Monteiro \\ Aguiar $^{4}$, José Barbosa dos Santos ${ }^{4}$, Paulo Roberto Cecon ${ }^{5}$
}

Resumo - Objetivou-se com este trabalho, avaliar os efeitos do sulfentrazone sobre as características associadas à atividade fotossintética de Helianthus annus, Canavalia ensiformis, Dolichos lab lab e Arachis hypogaea consideradas espécies com potencial de remediação de solo contaminado por esse herbicida. O experimento foi conduzido em esquema fatorial $4 \times 3 \mathrm{em}$ delineamento inteiramente casualizado, com quatro repetições. $\mathrm{O}$ primeiro fator constou do cultivo das espécies $H$. annus, $C$. ensiformis, D. lab lab e A. hypogaea e o segundo de três doses $\left(0,250\right.$ e $\left.500 \mathrm{~g} \mathrm{ha}^{-1}\right)$ de sulfentrazone aplicadas em pré-emergência. Após o preenchimento dos vasos com 6,0 kg de solo, classificado como Argissolo Vermelho-Amarelo, fez-se a irrigação e aplicou-se à superfície do solo o herbicida. Um dia após esta aplicação, procedeu-se a semeadura das espécies vegetais. Aos 40 dias após a emergência (DAE) das espécies vegetais, foram determinadas a condutância estomática $\left(\mathrm{gs}-\mathrm{mol} \mathrm{m}^{-1} \mathrm{~s}^{-1}\right)$, a taxa de transpiração ( $\mathrm{E}-\mathrm{mol} \mathrm{H}_{2} \mathrm{O} \mathrm{m}^{-2}$ $\left.\mathrm{s}^{-1}\right)$, a concentração de $\mathrm{CO}_{2}$ na câmara subestomática $\left(\mathrm{Ci}-\mu \mathrm{mol} \mathrm{mol}^{-1}\right)$, o gradiente de $\mathrm{CO}_{2}(\Delta \mathrm{C}$ -

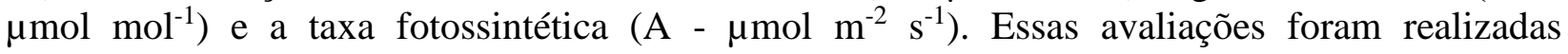
utilizando-se um analisador de gases no infravermelho (IRGA). Aos 100 DAE das plantas foi coletada a parte aérea e determinada a matéria seca. Resíduos de sulfentrazone no solo podem afetar as variáveis fisiológicas estudadas, todavia, a produção de matéria seca da parte aérea dessas espécies não foi influenciada pela ação do herbicida, independentemente das doses aplicadas, indicando a tolerância das espécies ao sulfentrazone.

Palavras-chaves: fitorremediação; herbicida; taxa fotossintética

\begin{abstract}
The goal of this work was to evaluate the sulfentrazone effects on the characteristics associated with photosynthetic activity of Helianthus annus, Canavalia ensiformis, Dolichos lab $l a b$ and Arachis hypogaea regarded species with potential of remediation of contaminated soil with this herbicide. The experiment was conducted in factorial $4 \times 3$, completely randomized design, with four replications. The first factor consisted by cultivation of species $H$. annus, $C$. ensiformis, D. lab lab and A. hypogaea; the second factor by three sulfentrazone doses $(0,250$ and $500 \mathrm{~g} \mathrm{ha}^{-1}$ ) applied in pre-emergence. After filling the pots with $6.0 \mathrm{~kg}$ of soil, classified with red-yellow Hapludalf, it was done the irrigation and it was done the herbicide application herbicide in soil surface. One day after this application, proceeded the sowing of vegetal species.

\footnotetext{
${ }^{1}$ Recebido para publicação em 03/03/2016 e aceito em 28/06/2016.

${ }^{2}$ Departamento de Agronomia, UFES. São Mateus, Espírito Santo, Brasil. E-mail: <ferreiragro@yahoo.com.br>.

${ }^{3}$ Departamento de Fitotecnia, UFV. Viçosa, Minas Gerais, Brasil. E-mail: <lroberto@ufv.br>.

${ }^{4}$ Departamento de Agronomia, UFVJM. Diamantina, Minas Gerais, Brasil. E-mail: <evanderalves@gmail.com, lumonaguiar@hotmail.com, jbarbosasantos@yahoo.com.br>.

${ }^{5}$ Departamento de Estatística, UFV. Viçosa, Minas Gerais, Brasil. E-mail: <cecon@ufv.br>.
} 
At 40 days after the emergency of the vegetal species, were evaluated the stomatal conductance (gs - mol m $\left.\mathrm{m}^{-1} \mathrm{~s}^{-1}\right)$, transpiration rate $\left(\mathrm{E}-\mathrm{mol} \mathrm{H}_{2} \mathrm{O} \mathrm{m}^{-2} \mathrm{~s}^{-1}\right)$, sub-stomatal $\mathrm{CO}_{2}$ concentration $(\mathrm{Ci}$ $\left.\mu \mathrm{mol} \mathrm{mol}{ }^{-1}\right), \mathrm{CO}_{2}$ gradient $\left(\Delta \mathrm{C}-\mu \mathrm{mol} \mathrm{mol}{ }^{-1}\right)$ and the photosynthetic rate $\left(\mathrm{A}-\mu \mathrm{mol} \mathrm{m} \mathrm{s}^{-1}\right)$. These evaluations were performed using an infrared gas analyzer (IRGA). At 100 days after the plants emergency was collected the shoot for determination of shoot dry matter. Sulfentrazone residues in soil can affect the physiological variables studied, however, the shoot dry matter wasn't influenced by the herbicide action, independently of the doses applied, indicating tolerance of the species to the sulfentrazone.

Keywords: phytoremediation; herbicide; photosynthetic rate

\section{Introdução}

O processo conhecido como fitorremediação consiste no método de descontaminação de solo ou água por meio da utilização de plantas. Estas devem, obrigatoriamente, ser tolerantes aos contaminantes e possuir habilidade para remover, extrair e/ou mesmo mineralizá-los no ambiente (Accioly e Siqueira, 2000). Ao longo dos últimos anos, foram realizados diversos trabalhos (Carmo et al., 2008; Procópio et al., 2008; Belo et al., 2011; Madalão et al., 2012) que visam a utilização dessa técnica em áreas contaminadas por agrotóxicos, como os herbicidas. Muitos desses produtos apresentam características que lhes permitem permanecer no ambiente por longos períodos.

A presença de resíduos de herbicidas no solo causa elevado impacto ambiental que pode ser responsável por perdas econômicas, quando afeta culturas sensíveis, além da redução na biodiversidade (Reis et al., 2008; Robinson, 2008). Somado a isso, também pode ocorrer a lixiviação desses herbicidas, os quais podem contaminar os cursos hídricos, e causar efeitos tóxicos em organismos aquáticos como fitoplânctons e peixes (Scheil et al., 2009).

Dentre os herbicidas que apresentam elevada persistência, e consequentemente, elevado potencial de contaminação ambiental, encontra-se o sulfentrazone, que é muito utilizado no Brasil nas culturas da cana-deaçúcar, soja, citrus, café, eucalipto e em áreas não agrícolas (Rodrigues e Almeida, 2011). Com isso, estudos sobre o comportamento desse herbicida no ambiente e de técnicas para reduzir sua persistência no ambiente são necessários. Uma das alternativas para descontaminação de solo com resíduos desse herbicida é a fitorremediação. Todavia, para viabilizar esse processo a primeira etapa é a identificação de plantas tolerantes a esse produto, que apresenta como mecanismo de ação a inibição da rota metabólica de síntese da enzima protoporfirinogênio oxidase (PROTOX), atuando indiretamente na síntese de clorofila em plantas sensíveis (Silva et al., 2007).

Ao inibir a enzima PROTOX, localizada nos cloroplastos, o sulfentrazone reduz a síntese das clorofilas, uma vez que essa enzima é a precursora das reações que transformam protoporfirinogênio IX em protoporfirina IX, e estes compostos são responsáveis pela formação das clorofilas. Com isso, ocorre o acúmulo de protoporfirinogênio IX e saída deste para o citoplasma, onde é oxidado formando a protoporfirina IX (Silva e Silva, 2007), esta que por sua vez interage com oxigênio e luz, formando oxigênio "singlet", uma espécie reativa de oxigênio (ROS), que desencadeia processos oxidativos como a peroxidação de lipídios das membranas (Tripathy et al., 2007).

$\mathrm{O}$ influxo de $\mathrm{CO}_{2}$ pode ser comprometido devido a atuação dos herbicidas inibidores da enzima PROTOX, sendo um dos fatores a formação de óxido nítrico por meio das ROS. O óxido nítrico estimula a síntese e atividade do ácido abscísico (ABA), hormônio que atua regulando o fechamento estomático (Taiz e Zeiger, 2013). Também, pode ocorrer o fechamento estomático pela ação das ROS 
favorecendo o acúmulo de cálcio no citossol (Taiz e Zeiger, 2013), ou ainda devido à peroxidação das membranas celulares das células adjacentes aos estômatos.

O sulfentrazone causa redução da fotossíntese devido a menor síntese de clorofilas, que são proteínas que apresentam função vital, capturando a energia luminosa, que será convertida em poder redutor para o processo de fixação e assimilação do $\mathrm{CO}_{2}$ no ciclo de Calvin (Carretero, 2008). Além disso, as clorofilas podem sofrer os danos causados pelas ROS (Gan, 2007), reduzindo ainda mais a atividade fotossintética. À medida que aumenta o estresse oxidativo em função do tempo de exposição à luz, os tilacóides são danificados e perdem sua capacidade de realizar fotossíntese, devido a danos na maquinaria fotossintética (Tripathy et al., 2007).

Diante do exposto, objetivou-se, com este trabalho, avaliar as características relacionadas à atividade fotossintética das espécies Helianthus annus, Canavalia ensiformis, Dolichos lab lab e Arachis hypogaea quando cultivadas em solo contaminado com diferentes concentrações de sulfentrazone.

\section{Material e Métodos}

O trabalho foi conduzido em casa de vegetação da Universidade Federal de Viçosa, Viçosa (MG). O experimento constou de um fatorial $4 \times 3$, no delineamento inteiramente casualizado, com quatro repetições. $\mathrm{O}$ primeiro fator foi constituído pelo cultivo das espécies Heliantum annus, Canavalia ensiformis, Dolichos lab lab e Arachis hypogaea e o segundo de três doses $\left(0,250\right.$ e $\left.500 \mathrm{~g} \mathrm{ha}^{-1}\right)$ de sulfentrazone, aplicadas em pré-emergência.

Como substrato para o crescimento das plantas, utilizou-se amostras de solo classificado como Argissolo VermelhoAmarelo, cujas características físicas e químicas estão apresentadas na Tabela 1. As amostras do solo em estudo foram coletadas na profundidade de 0-20 cm, em área sem histórico de aplicação de herbicidas. Essas foram passadas em peneira com malha de 4 mm e adubadas com superfosfato simples na proporção de $10 \mathrm{~g} \mathrm{~kg}^{-1}$ de solo.

Tabela 1. Resultados das análises física e química das amostras do Argissolo Vermelho-Amarelo utilizado no experimento.

\begin{tabular}{|c|c|c|c|c|c|c|c|c|}
\hline \multicolumn{9}{|c|}{ Análise Física $\left({\left.\text { dag } \mathrm{kg}^{-1}\right)^{1}}^{1}\right.$} \\
\hline Argila & Silte & Areia fina & \multicolumn{3}{|c|}{ Areia grossa } & \multicolumn{3}{|c|}{ Classificação textural } \\
\hline 24 & 20 & 31 & \multicolumn{3}{|c|}{25} & \multicolumn{3}{|c|}{ Franco Argilo Arenosa } \\
\hline \multicolumn{9}{|c|}{ Análise Química $^{1}$} \\
\hline $\mathrm{pH}$ & $\mathrm{K}^{+}$ & $\mathrm{H}+\mathrm{Al}$ & $\mathrm{Ca}^{2+}$ & $\mathrm{Mg}^{2+}$ & $\mathrm{CTC}_{(\mathrm{pH} 7,0)}$ & $\mathrm{V}$ & $\mathrm{m}$ & MO \\
\hline $\mathrm{H}_{2} \mathrm{O}$ & $\mathrm{mg} \mathrm{dm}^{-3}$ & \multicolumn{4}{|c|}{$\mathrm{cmol}_{\mathrm{c}} \mathrm{dm}^{-3}$} & \multicolumn{2}{|c|}{$\%$} & dag $\mathrm{kg}^{-1}$ \\
\hline 5,0 & 49,7 & 3,63 & 5,9 & 1,5 & 11,34 & 68 & 1 & 1,3 \\
\hline
\end{tabular}

${ }^{1}$ Análises realizadas no Laboratório de Análise de Solos Viçosa Ltda.

Após o preparo do solo, o mesmo foi colocado em vasos revestidos com filme de polietileno visando evitar perda do herbicida por lixiviação. Foi utilizado $6,0 \mathrm{~kg}$ do substrato por vaso, estes foram irrigados ajustando-se a umidade em valor próximo a $80 \%$ da capacidade de campo, fazendo-se a seguir a aplicação do herbicida com um pulverizador de precisão, equipado com bicos TT110.02, espaçados a $0,5 \mathrm{~m}$, calibrado para aplicação de $100 \mathrm{~L} \mathrm{ha}^{-1}$ de calda herbicida.

A semeadura das espécies $H$. annus, $C$. ensiformis, $D$. lab lab e A. hypogaea foi realizada um dia após a aplicação do sulfentrazone. Sete dias após a emergência foi realizado o desbaste, deixando três plantas por vaso. Foram feitas irrigações diárias, mantendo-se a umidade do solo próxima a $80 \%$ da capacidade de campo. O controle da 
umidade do solo foi realizado por meio de pesagens semanais dos vasos repondo-se a água evapotranspirada.

Aos 30, 45 e 60 dias após emergência (DAE) foi adicionado em cada vaso $0,3 \mathrm{~g}$ de uréia visando melhor crescimento das plantas. A partir dos 60 DAE foram feitas adubações semanais de cobertura com $100 \mathrm{~mL}$ por vaso de solução Ouro Verde ${ }^{\circledR}$ contendo $\left(\mathrm{g} \mathrm{L}^{-1}\right)$ : N $(3,75), \mathrm{P}_{2} \mathrm{O}_{5}(3,75), \mathrm{K}_{2} \mathrm{O}(5,00), \mathrm{CaO}(0,75), \mathrm{S}$ $(1,00), \mathrm{MgO}(0,20), \mathrm{Cl}(0,10), \mathrm{Mn}(0,075), \mathrm{Fe}$ $(0,015), \mathrm{B}(0,012)$ e $\mathrm{Zn}(0,010)$.

Aos 40 DAE das plantas foram realizadas as avaliações na folha mais jovem, com limbo foliar totalmente expandido, das quatro espécies, utilizando-se analisador de gases no infravermelho (IRGA), marca ADC, modelo LCA PRO (Analytical Development Co. Ltd, Hoddesdon, UK). Foram determinadas a condutância estomática ( $\left.\mathrm{gs}-\mathrm{mol} \mathrm{m}^{-1} \mathrm{~s}^{-1}\right)$, a taxa de transpiração $\left(\mathrm{E}-\mathrm{mol} \mathrm{H}_{2} \mathrm{O} \mathrm{m} \mathrm{m}^{-2} \mathrm{~s}^{-1}\right)$, a concentração de $\mathrm{CO}_{2}$ na câmara subestomática $\left(\mathrm{Ci}-\mu \mathrm{mol} \mathrm{mol}{ }^{-1}\right)$, o gradiente de $\mathrm{CO}_{2}(\Delta \mathrm{C}$ $\left.\mu \mathrm{mol} \mathrm{mol}^{-1}\right)$ e a taxa fotossintética (A - $\mu \mathrm{mol}$ $\left.\mathrm{m}^{-2} \mathrm{~s}^{-1}\right)$. Essas avaliações foram realizadas entre 7 e 9 horas da manhã, em dia de céu limpo e com iluminação artificial de 1.200 $\mu \mathrm{mol} \mathrm{m} \mathrm{m}^{-2} \mathrm{~s}^{-1}$, de forma a manter as condições ambientais homogêneas durante as avaliações em casa de vegetação aberta, permitindo livre circulação do ar.

Aos 100 DAE fez-se a colheita da parte aérea das plantas, que foram secas em estufa de circulação forçada de ar $\left(70 \pm 1^{\circ} \mathrm{C}\right)$ até peso constante, determinando-se a matéria seca.

Os dados foram submetidos à análise de variância e de regressão, comparando somente o fator doses. Os modelos foram escolhidos com base na significância dos coeficientes de regressão, utilizando-se o teste " $t$ " a $5 \%$ de probabilidade, no fenômeno biológico e no coeficiente de determinação $\left(r^{2}=\right.$ SQReg/SQTrat).

\section{Resultados e Discussão}

Observou-se redução da condutância estomática (gs) nas plantas de $H$. annus, $C$. ensiformis, D. lab lab e A. hypogaea quando estas espécies foram cultivadas em solo tratado com sulfentrazone, apresentando os menores valores com a maior concentração do herbicida no solo (Tabela 2). Esse herbicida induz a produção de espécies reativas de oxigênio (ROS), estas que por sua vez atuam como mensageiros secundários na ativação de canais da membrana plasmática, possibilitando o influxo de cálcio, que se concentra no citossol causando fechamento estomático (Taiz e Zeiger, 2013), reduzindo assim a condutância estomática. As ROS, também, podem promover a formação de óxido nítrico, o qual estimula a síntese e atividade do ácido abscísico, hormônio que atua regulando o fechamento estomático (Taiz e Zeiger, 2013)

Tabela 2. Condutância estomática (gs) de espécies fitorremediadoras, cultivadas por 40 dias em solo tratado ou não com sulfentrazone, com as respectivas equações de regressão e coeficientes de determinação.

\begin{tabular}{|c|c|c|c|c|c|}
\hline \multirow{3}{*}{ Espécies fitorremediadoras } & \multicolumn{3}{|c|}{ gs $\left(\mathrm{mol} \mathrm{m}^{-1} \mathrm{~s}^{-1}\right)$} & \multirow{3}{*}{ Equação de regressão } & \multirow{3}{*}{$r^{2}$} \\
\hline & \multicolumn{3}{|c|}{ Doses $\left(\mathrm{g} \mathrm{ha}^{-1}\right)$} & & \\
\hline & 0 & 250 & 500 & & \\
\hline Helianthus annus & 5,97 & 4,23 & 2,75 & $\hat{\mathrm{Y}}=5,9300-0,0065 * \mathrm{D}$ & 0,693 \\
\hline Canavalia ensiformis & 3,66 & 3,16 & 1,21 & $\hat{\mathrm{Y}}=3,8983-0,0049 * \mathrm{D}$ & 0,608 \\
\hline Dolichos lab lab & 0,99 & 1,19 & 0,28 & $\hat{\mathrm{Y}}=1,1746-0,0014 * \mathrm{D}$ & 0,502 \\
\hline Arachis hypogaea & 1,66 & 1,04 & 0,71 & $\hat{Y}=1,6088-0,0019 * D$ & 0,489 \\
\hline C.V. (\%) & & 31,16 & & & \\
\hline
\end{tabular}

${ }^{*}$ Significativo a $5 \%$ pelo teste $\mathrm{t}$. 
A condutância estomática é proporcional ao número, tamanho e diâmetro de abertura dos estômatos e características que dependem de outros fatores endógenos e ambientais (Brodribb e Holbrook, 2003). Entretanto, em condições de estresse a planta tende a fechar os estômatos como mecanismo de defesa contra a perda de água, aumentando a resistência e por consequência reduzindo a condutância estomática (Taiz e Zeiger, 2013).

A redução da condutância estomática foi observada em soja e Portulaca oleracea 6 horas após a aplicação do lactofen, um herbicida inibidor da enzima PROTOX (Wichert e Talbert, 1993). Esse herbicida pode promover o fechamento estomático devido aos processos oxidativos e aumento da concentração de óxido nítrico que atuam como sinalizadores para o ABA, hormônio que atua regulando o fechamento estomático (Taiz e
Zeiger, 2013) Esta atuação do óxido nítrico tem sido observada em plantas de soja após a aplicação de lactofen (Carretero, 2008).

A taxa de transpiração (E) das espécies H. annus e A. hypogaea não apresentou alteração, independentemente das concentrações de sulfentrazone no solo (Tabela 3). No entanto, as espécies $C$. ensiformis e $D$. lab lab apresentaram correlação negativa dessa variável com a concentração do sulfentrazone no solo, havendo redução do valor da E com o aumento da concentração deste herbicida no solo. A menor E está associada ao fechamento dos estômatos o que leva, em consequência, a uma limitação da taxa de fotossíntese. (Silva et al., 2010), porém, nem sempre a diminuição da E está relacionado ao estresse hídrico, pode ser um mecanismo da planta para manter a turgidez nos períodos de maior perda de vapor de água para a atmosfera (Casaroli et al. 2008).

Tabela 3. Taxa de transpiração (E) de espécies fitorremediadoras, cultivadas por 40 dias em solo tratado ou não com sulfentrazone, com as respectivas equações de regressão e coeficientes de determinação.

\begin{tabular}{|c|c|c|c|c|c|}
\hline \multirow{3}{*}{ Espécies fitorremediadoras } & \multicolumn{3}{|c|}{$\mathrm{E}\left(\mathrm{mol} \mathrm{H}_{2} \mathrm{O} \mathrm{m}^{-2} \mathrm{~s}^{-1}\right)$} & \multirow{3}{*}{ Equação de regressão } & \multirow{3}{*}{$r^{2}$} \\
\hline & \multicolumn{3}{|c|}{ Doses $\left(\mathrm{g} \mathrm{ha}^{-1}\right)$} & & \\
\hline & 0 & 250 & 500 & & \\
\hline Helianthus anпиs & 4,22 & 4,16 & 4,30 & $\hat{\mathrm{Y}}=4,22$ & - \\
\hline Canavalia ensiformis & 5,63 & 5,65 & 5,09 & $\hat{\mathrm{Y}}=5,7242-0,0011 * \mathrm{D}$ & 0,431 \\
\hline Dolichos lab lab & 4,70 & 4,95 & 4,05 & $\hat{\mathrm{Y}}=4,8925-0,0013 * \mathrm{D}$ & 0,277 \\
\hline Arachis hypogaea & 4,51 & 4,31 & 4,38 & $\hat{\mathrm{Y}}=4,40$ & - \\
\hline C.V. (\%) & & 5,83 & & & \\
\hline
\end{tabular}

${ }^{*}$ Significativo a $5 \%$ pelo teste $\mathrm{t}$.

Para H. annus não foi observada diferenças na concentração de $\mathrm{CO}_{2}$ na câmara subestomática $(\mathrm{Ci})$ independentemente das concentrações do herbicida no solo (Tabela 4). No entanto, para $C$. ensiformis, D. lab lab e A. hypogaea foi observado correlação negativa da $\mathrm{Ci}$ com a concentração do sulfentrazone no solo, havendo redução do valor da $\mathrm{Ci}$ com o aumento da concentração. Esses resultados demonstram haver menor influxo de $\mathrm{CO}_{2}$ para o espaço subestomático com aumento da concentração do herbicida no solo.

A redução da $\mathrm{Ci}$ é ocasionada, geralmente, por fatores que reduzem o influxo de $\mathrm{CO}_{2}$ para o espaço interno das folhas devido à redução da condutância estomática. Isso, geralmente, ocorre pelo fechamento dos estômatos que são influenciados por alguns fatores ambientais como disponibilidade hídrica, luz e energia, entre outros (Ometto et al., 2003). Essa variável fisiológica, também, pode ser influenciada pelas ROS, estas que atuam como mensageiros secundários na ativação de canais da membrana plasmática, possibilitando o influxo de cálcio, que se concentra no citossol causando fechamento estomático (Taiz e Zeiger, 2013), reduzindo 
assim a condutância estomática (menor influxo de $\mathrm{CO}_{2}$ ).

O controle estomático é importante propriedade fisiológica, por meio da qual, as plantas limitam a perda de água, ocasionando reduções na condutância estomática e, geralmente, reduzindo as trocas gasosas como forma de resposta das plantas a diversos fatores, incluindo o estresse hídrico (Paiva et al., 2005).

Tabela 4. Concentração de $\mathrm{CO}_{2}$ na câmara subestomática $(\mathrm{Ci})$ de espécies fitorremediadoras, cultivadas por 40 dias em solo tratado ou não com sulfentrazone, com as respectivas equações de regressão e coeficientes de determinação.

\begin{tabular}{|c|c|c|c|c|c|}
\hline \multirow{3}{*}{ Espécies fitorremediadoras } & \multicolumn{3}{|c|}{$\mathrm{Ci}\left(\mu \mathrm{mol} \mathrm{mol}{ }^{-1}\right)$} & \multirow{3}{*}{ Equação de regressão } & \multirow{3}{*}{$r^{2}$} \\
\hline & \multicolumn{3}{|c|}{ Doses $\left(\mathrm{g} \mathrm{ha}^{-1}\right)$} & & \\
\hline & 0 & 250 & 500 & & \\
\hline Helianthus annus & 240,00 & 239,50 & 234,25 & $\hat{Y}=237,92$ & - \\
\hline Canavalia ensiformis & 227,25 & 239,00 & 211,00 & $\hat{\mathrm{Y}}=239,750-0,056 * \mathrm{D}$ & 0,596 \\
\hline Dolichos lab lab & 229,25 & 232,25 & 186,75 & $\hat{\mathrm{Y}}=238,833-0,091 * \mathrm{D}$ & 0,707 \\
\hline Arachis hypogaea & 201,68 & 193,00 & 181,25 & $\hat{\mathrm{Y}}=202,187-0,041 * \mathrm{D}$ & 0,637 \\
\hline C.V. $(\%)$ & & 4,16 & & & \\
\hline
\end{tabular}

${ }^{*}$ Significativo a $5 \%$ pelo teste $\mathrm{t}$.

Galon et al., (2010), trabalhando com a aplicação de ametryn, verificaram que a concentração de $\mathrm{CO}_{2}$ no mesófilo foliar foi por volta de $50 \%$ maior nas plantas sob ação do herbicida ametryn do que na testemunha sem aplicação, considerando que este produto atua diretamente na fotossíntese (fotossistema II). Já o sulfentrazone, que também inibe a fotossíntese de forma indireta, apresenta efeitos diversos, isso devido a menor síntese de pigmentos. A redução no teor de pigmentos devido à ação de inibidores da PROTOX foi observada por outros autores (Tripathy et al.,
2007). Esses efeitos na fotossíntese são intensificados pela ação das ROS, que leva a redução da maquinaria fotossintética.

Para as plantas de $H$. annus, $C$. ensiformis e A. hypogaea observou-se redução no gradiente de $\mathrm{CO}_{2}(\Delta \mathrm{C})$, quando essas espécies foram cultivadas em solo tratado com sulfentrazone em relação ao tratamento sem herbicida, com menores valores com aumento da concentração deste herbicida no solo. Já para $D$. lab lab não houve redução do $\Delta \mathrm{C}$ independentemente da dose de sulfentrazone aplicada (Tabela 5).

Tabela 5. Gradiente de $\mathrm{CO}_{2}(\Delta \mathrm{C})$ de espécies fitorremediadoras, cultivadas por 40 dias em solo tratado ou não com sulfentrazone, com as respectivas equações de regressão e coeficientes de determinação.

\begin{tabular}{|c|c|c|c|c|c|}
\hline \multirow{3}{*}{ Espécies fitorremediadoras } & \multicolumn{3}{|c|}{$\Delta \mathrm{C}\left(\mu \mathrm{mol} \mathrm{mol}^{-1}\right)$} & \multirow{3}{*}{ Equação de regressão } & \multirow{3}{*}{$r^{2}$} \\
\hline & \multicolumn{3}{|c|}{ Doses $\left(\mathrm{g} \mathrm{ha}^{-1}\right)$} & & \\
\hline & 0 & 250 & 500 & & \\
\hline Helianthus annus & 114,75 & 96,75 & 101,75 & $\hat{\mathrm{Y}}=113,417-0,036 * \mathrm{D}$ & 0,877 \\
\hline Canavalia ensiformis & 109,50 & 110,00 & 100,75 & $\hat{\mathrm{Y}}=111,125-0,018 * \mathrm{D}$ & 0,535 \\
\hline Dolichos lab lab & 80,25 & 83,00 & 74,25 & $\hat{\mathrm{Y}}=79,17$ & - \\
\hline Arachis hypogaea & 125,00 & 120,75 & 83,00 & $\hat{\mathrm{Y}}=130,583-0,084 * \mathrm{D}$ & 0,801 \\
\hline C.V. (\%) & & 2,96 & & & \\
\hline
\end{tabular}

${ }^{*}$ Significativo a $5 \%$ pelo teste $t$.

A redução do $\Delta \mathrm{C}$ foi observada em cana-de-açúcar após a aplicação do ametryn, que atua diretamente na fotossíntese (Galon et al., 2010). Segundo esses autores, o $\Delta \mathrm{C}$ está diretamente relacionado à intensidade fotossintética da planta no momento da 
avaliação, ou seja, quanto mais lento for o metabolismo da planta, menor o consumo de $\mathrm{CO}_{2}$ por unidade de tempo, reduzindo a diferença entre a concentração de carbono da atmosfera e do espaço interno da folha $(\Delta C)$. Como o sulfentrazone atua inibindo a atividade fotossintética, mesmo de forma indireta, os efeitos tendem a ser semelhantes.

As plantas de $H$. annus apresentaram redução da taxa fotossintética (A) quando cultivadas em solos contaminado com sulfentrazone, com maiores reduções nas maiores concentrações do herbicida (Tabela 6). Para essa espécie não foi observada influencia do herbicida na Ci (Tabela 4), evidenciando que a fotossíntese provavelmente não foi reduzida pela deficiência do substrato $\mathrm{CO}_{2}$. Para D. lab lab e A. hypogaea a taxa fotossintética, também, foi proporcionalmente reduzida com aumento da concentração de sulfentrazone no solo. Houve redução de aproximadamente $21 \%$ e $34 \%$ para $D$. lab lab e A. hypogaea respectivamente, ao se comparar a dose de $500 \mathrm{~g} \mathrm{ha}^{-1}$ do sulfentrazone com a ausência deste herbicida. No entanto, para $C$. ensiformis não foi observada influência na atividade fotossintética por nenhuma das doses testadas do herbicida (Tabela 6).

Tabela 6. Taxa fotossintética (A) de espécies fitorremediadoras, cultivadas por 40 dias em solo tratado ou não com sulfentrazone, com as respectivas equações de regressão e coeficientes de determinação.

\begin{tabular}{|c|c|c|c|c|c|}
\hline \multirow{3}{*}{ Espécies fitorremediadoras } & \multicolumn{3}{|c|}{$\mathrm{A}\left(\mu \mathrm{mol} \mathrm{m} \mathrm{m}^{-2} \mathrm{~s}^{-1}\right)$} & \multirow{3}{*}{ Equação de regressão } & \multirow{3}{*}{$\mathrm{r}^{2}$} \\
\hline & \multicolumn{3}{|c|}{ Doses $\left(\mathrm{g} \mathrm{ha}^{-1}\right)$} & & \\
\hline & 0 & 250 & 500 & & \\
\hline Helianthus annus & 35,66 & 31,03 & 31,66 & $\hat{\mathrm{Y}}=34,780-0,008 * \mathrm{D}$ & 0,445 \\
\hline Canavalia ensiformis & 31,79 & 35,09 & 34,15 & $\hat{Y}=33,68$ & - \\
\hline Dolichos lab lab & 28,38 & 26,92 & 22,46 & $\hat{\mathrm{Y}}=28,878-0,012 * \mathrm{D}$ & 0,477 \\
\hline Arachis hypogaea & 41,22 & 39,62 & 27,18 & $\hat{\mathrm{Y}}=43,026-0,028 * \mathrm{D}$ & 0,741 \\
\hline C.V. $(\%)$ & & 6,73 & & & \\
\hline
\end{tabular}

${ }^{*}$ Significativo a $5 \%$ pelo teste $\mathrm{t}$

Considerando o mecanismo de ação dos herbicidas inibidores da PROTOX, a atividade fotossintética é comprometida por alguns distúrbios relacionados com a peroxidação de lipídios e pigmentação foliar, pois os herbicidas desse grupo atuam na redução dos teores de clorofila e carotenóides foliares, efeitos estes que podem ser observados também em plantas tolerantes (Carretero, 2008). Esses herbicidas podem atuar também na liberação de etileno, etano e aldeído malônico (Kenyon et al. 1985), que podem influenciar no balaço hormonal, e consequentemente em várias atividades fisiológicas das plantas.

Os efeitos provocados pelo sulfentrazone podem ser observados até mesmo em plantas consideradas tolerantes, como é o caso da soja, em que foi observado extravasamento de eletrólitos e outros danos celulares, com maiores efeitos tanto com aumento da luminosidade quanto da concentração do inibidor (Li et al., 2000).

Com a redução, especialmente, dos teores de carotenóides há dificuldade na dissipação de energia (Tripathy et al., 2007), levando a um estresse oxidativo, causando destruição de membranas e redução da maquinaria fotossintética. Também, há peroxidação dos lipídios provocado pelo oxigênio "singlet" gerado pela protoporfirina IX no citoplasma (Weller, 2003). A redução nos teores de clorofila total e de carotenóides foi observada em folhas de Toona ciliata tratadas com sulfentrazone nas doses $600 \mathrm{e}$ $1.200 \mathrm{~g} \mathrm{ha}^{-1}$ (Oliveira et al. 2008).

$\mathrm{O}$ acúmulo de matéria seca da parte aérea das plantas de $H$. annus, $C$. ensiformis, $D$. lab lab e A. hypogaea não foi influenciado pela ação do sulfentrazone, independentemente 
das doses aplicadas (Tabela 7), comprovando a tolerância dessas espécies ao herbicida, que é uma das características altamente desejável para que uma espécie seja utilizada como fitorremediadora. Dessa forma, $H$. annus, $C$. ensiformis, $D$. lab lab e A. hypogaea apresentam potencial para fitorremediação de solos tratados com sulfentrazone.

Pode-se inferir que as plantas com ação fitorremediadora de um determinado herbicida apresentam tolerância do mesmo, em função dos aspectos fisiológicos relacionados com os mecanismos de desintoxicação, tais como metabolização, translocação diferenciada, compartimentalização do produto e impedimentos anatômicos a penetração ou absorção do produto, no entanto esses mecanismos podem não apresentar tanta eficiência na proteção das plantas, como observado por Carretero (2008) em plantas de soja.

Tabela 7. Matéria seca da parte aérea (MSPA) de espécies fitorremediadoras, cultivadas por 100 dias em solo tratado ou não com sulfentrazone, com as respectivas equações de regressão.

\begin{tabular}{|c|c|c|c|c|}
\hline \multirow{3}{*}{ Espécies fitorremediadoras } & \multicolumn{3}{|c|}{$\operatorname{MSPA}(\mathrm{g})$} & \multirow{3}{*}{ Equação de regressão } \\
\hline & \multicolumn{3}{|c|}{ Doses $\left(\mathrm{g} \mathrm{ha}^{-1}\right)$} & \\
\hline & 0 & 250 & 500 & \\
\hline Helianthus annus & 73,00 & 65,00 & 65,00 & $\hat{\mathrm{Y}}=67,67$ \\
\hline Canavalia ensiformis & 156,00 & 173,00 & 173,00 & $\hat{\mathrm{Y}}=167,33$ \\
\hline Dolichos lab lab & 107,00 & 126,00 & 126,00 & $\hat{Y}=119,67$ \\
\hline Arachis hypogaea & 50,00 & 61,00 & 61,00 & $\hat{Y}=57,33$ \\
\hline C.V. (\%) & & 15,58 & & \\
\hline
\end{tabular}

${ }^{*}$ Significativo a $5 \%$ pelo teste $\mathrm{t}$.

\section{Conclusões}

Foi possível observar alteração da atividade fotossintética nas espécies estudadas na presença do herbicida, sendo que a intensidade desse efeito variou com a espécie vegetal. Todavia, a produção de matéria seca da parte aérea não foi influenciada pelo sulfentrazone indicando que as espécies são tolerantes a esse herbicida.

\section{Agradecimentos}

CAPES, CNPq e FAPEMIG.

\section{Referências}

Accioly, A.M.A.; Siqueira, J.O. Contaminação química e biorremediação do solo. In: Novais RF, Alvarez VVH, Schaefer CEGR (Ed.). Tópicos em ciência do solo. Viçosa: Sociedade Brasileira de Ciência do Solo, 2000. v.1. p. 299-352.

Belo, A.F.; Coelho, A.T.C.P.; Ferreira, L.R.; Silva A.A.; Santos, J.B. Potencial de espécies vegetais na remediação de solo contaminado com sulfentrazone. Planta Daninha, v.29, n.4, p.821-828, 2011.

Brodribb, T.J.; Holbrook, N.M. Stomatal closure during leaf dehydration, correlation with other leaf physiological traits. Plant Physiology, v.132, n.4, p.2166-2173, 2003.

Carmo, M.L.; Procópio, S.O.; Pires, F.R.; Cargnelutti Filho, A.; Braz, G.B.P.; Silva, W.F.P. et al. Influência do período de cultivo de Panicum maximum (Cultivar Tanzânia) na fitorremediação de solo contaminado com picloram. Planta Daninha, v.26, n.2, p.315322, 2008

Carretero, D.M. Efeitos da inibição da protoporfirinogênio IX oxidase sobre as trocas gasosas e fluorescência da clorofila a em plantas de soja (Glycine max L. Merrill). 2008. 57f. Dissertação (Mestrado em Fisiologia Vegetal) - Universidade Federal de Viçosa, Viçosa, 2008. 
Cesaroli, D.; Lier, Q.J.V.; Irigoyen, A.I.; Simon, J.; Scarpare, F.V. Estimativa da taxa de transpiração do feijoeiro a partir de medidas de porometria, em diferentes camadas do dossel. Revista Brasileira de Agrometeorologia, v.16, n.2, p.149-153, 2008.

Galon, L.; Ferreira, F.A.; Silva, A.A.; Concenço, G.; Ferreira, E.A.; Barbosa, M.H.P. et al. Influência de herbicidas na atividade fotossintética de genótipos de cana-de-açúcar. Planta Daninha, 2010. v.28, n.3. p.591-597.

Gan, S. Senescende processes in plants. Blackwell Publishing Ltd, Iowa, USA, 2007. p.322.

Gove, B.; Power, S.A.; Buckley, G.P.; Ghazoul, J. Effects of herbicide spray drift and fertilizer overspread on selected species of woodland ground flora: comparison between short-term and long-term impact assessments and field surveys. Journal of Applied Ecololy, v.44, n.2, p.374-384, 2007.

Kenyon, W.H.; Duke, S.O. Effects of acifluorfen on endogenous antioxidants and protective enzymes in cucumber (Cucumis sativus L.) cotyledons. Plant Physiology, v.79, n.3, p.862-866, 1985

Li, Z.; Walker R.H.; Wehtje G.; Hancock HG. Using electrolyte leakage to detect soybean (Glycine max) cultivars sensitive to sulfentrazone. Weed Technology, v.14, n.4, p.699-704, 2000.

Madalão, J.C.; Pires, F.R.; Chagas, K.; Cargnelutti Filho, A.; Procópio, S.O. Uso de leguminosas na fitorremediação de solo contaminado com sulfentrazone. Pesquisa Agropecuária Tropical, v.42, n.4, p.390-396, 2012.

Oliveira, J.R.; Duarte, N.F.; Fassio, P.O. Análise dos teores de clorofila e carotenóides como indicadores de fitotoxicidade de herbicidas em Toona ciliata var. australis. In: I JORNADA CIENTÍFICA e VI FIPA do CEFET Bambuí. Anais... Bambuí/MG - 2008.
Ometto, J.P.H.B.; Ehleringer, J.R.; Martinelli, L.A.; Berry, J.; Flanagan, L.; Domingues, T.F. et al. Variação temporal do isótopo estável do carbono em material arbóreo em florestas da região Amazônica. In: CONGRESSO BRASILEIRO DE ECOLOGIA, 4., 2003, Fortaleza. Anais... Rio Claro: Sociedade de Ecologia do Brasil, 2003.

Paiva, A.S.; Fernandes, E.J; Rodrigues, T.J.D.; Turco J.E.P. Condutância estomática em folhas de feijoeiro submetido a diferentes regimes de irrigação. Engenharia Agrícola, v.25, n.1, p.161-169, 2005.

Procópio, S.O.; Carmo, M.L.; Pires, F.R.; Cargnelutti Filho, A.; Braz, G.B.P.; Silva, W.F.P et al. Fitorremediação de solo contaminado com picloram por capim-pé-degalinha-gigante (Eleusine coracana). Revista Brasileira de Ciência do Solo, v.32, n.6, p.2517-2524, 2008.

Reis, M.R.; Silva, A.A.; Costa, M.D.; Guimarães, A.A.; Ferreira, E.A.; Santos, J.B. et al. Atividade microbiana em solo cultivado com cana-de-açúcar após aplicação de herbicidas. Planta Daninha, v.26, n.2, p.323331, 2008.

Robinson, D.E. Atrazine accentuates carryover injury from mesotrione in vegetable crops. Weed Technology, v.22, n.4, p.641-645, 2008.

Rodrigues B.N.; Almeida F.S. Guia de herbicidas. 6.ed. Londrina: 2011. 697 p.

Scheil, V.; Kienle, C.; Osterauer, R.; Kohler, H. Effects of 3,4-dichloroaniline and diazinon on different biological organisation levels of zebrafish (Danio rerio) embryos and larvae. Ecotoxicology, v.18, n.3, p.355-363, 2009.

Silva, A.A.; Silva, J.F. Competição entre plantas daninhas e culturas. In: Silva, A.A.; Silva, J.F. (Eds.). Tópicos em manejo de plantas daninhas. Viçosa, MG: Sociedade Brasileira de Ciência do Solo, 2007. p.17-61.

Silva, A.A.; Ferreira, F.A.; Ferreira, L.R.; Santos, J.B. Herbicidas: Classificação e 
mecanismo de ação. In: Silva, A.A.; Silva, J.F. (Eds.). Tópicos em manejo de plantas daninhas. Viçosa: Ed. UFV, 2007. p.63-81.

Silva, C.D.S.; Santos, P.A.A.; Lira, J.M.S.; Santana, M.C.; Silva Junior, C.D. Curso diário das trocas gasosas em plantas de feijão-caupi submetidas à deficiência hídrica. Revista Caatinga, v.23, n.4, p.7-13, 2010.

Taiz, L.; Zeiger, E. Fisiologia vegetal. Porto Alegre: Artmed, 2013. 954p.

Tripathy, B.C.; Mohapatra, A.; Gupta, I. Impairment of the photosynthetic apparatus by oxidase stress induced by photosensitization reaction of protoporphyrin IX. Biochimica et Biophysica Acta, v.1767, n.6, p.860-868, 2007.

Weller, S. Principles of selective weed control with herbicides. In: Herbicide action course. West Lafayette: Purdue University, 2003. p.101-130.

Wichert, R.A.; Talbert, R.E. Soybean (Glycine $\max$ L.) response to lactofen. Weed Science, v.41, n.1, p.23-27, 1993. 